\section{State of the ablation nation: a review of ablative therapies for cure in the treatment of hepatocellular carcinoma}

\author{
Umer Salati ${ }^{1}$, Aisling Barry², Frank Y Chou' ${ }^{1}$ Roy Ma² \& David M Liü,1
}

Primary liver cancer, mainly hepatocellular carcinoma, is one of the most common malignancies worldwide. Surgical management, either resection or transplantation, is considered definitive treatment, however, less than $20 \%$ of patients are ultimately candidates. Thermal ablation modalities such as radiofrequency ablation and microwave ablation have evolved such that these modalities have been applied with curative intent. Moreover, thermal ablation has demonstrated efficacy in treating early-stage tumors and can be offered as first-line treatment in patients with uncomplicated disease. Attributing to refinements in technology and techniques, recent studies evaluating stereotactic ablative body radiotherapy have shown promising results, while irreversible electroporation, an emerging modality, may further expand the role of ablative therapy in treating potentially resectable hepatocellular carcinoma.

First draft submitted: 9 February 2017; Accepted for publication: 28 March 2017; Published online: 7 July 2017

Primary liver cancer, mainly hepatocellular carcinoma (HCC), is one of the most common malignancies in the world, numbering over 700,000 new cases annually [1]. In men, HCC ranks fifth in the number of new cases annually and second in annual cancer-related deaths [2]. HCC demonstrates a high association with $\mathrm{HBV}$ and $\mathrm{HCV}$, alcohol consumption, aflatoxin $\mathrm{B} 1$ and cirrhosis [3]. Liver transplantation or local resection are considered first-line treatments in suitable patients, with the choice of surgery traditionally based upon the Milan criteria $[4,5]$ and liver function, as defined by bilirubin levels and either hepatic venous pressure gradient or platelet count [5]. However, only a small proportion of patients $(5-10 \%)$ meet the criteria for resection and while transplantation is essentially curative, a shortage of donors leading to significant delay ( $>6$ months) to transplant, in addition to cost, can result in limited access and impaired effectiveness [6,7]. Furthermore, patients who undergo liver resection may continue to harbor the underlying inflammatory processes, thus resulting in metachronous presentation of disease, creating a secondary population in need of continued surveillance and therapy.

Radiofrequency ablation (RFA), a thermal ablative modality, has been the mainstay of treatment offered to patients who are not suitable for resection or do not meet criteria for transplantation. The relatively newer microwave ablation (MWA), another thermal technique has increased in popularity and serves as an alternative to RFA, with a lesser supporting body of evidence and expanded indications (location and size) as compared with RFA. Recently, external beam radiotherapy, specifically

'Department of Radiology, Vancouver General Hospital, Vancouver, BC, Canada

2Department of Radiation Oncology, BC Cancer Agency, Vancouver, BC, Canada

*Author for correspondence: Dave.Liu@vch.ca

\section{KEYWORDS}

- hepatocellular carcinoma

- primary liver tumor

- irreversible electroporation

- microwave ablation

- radiofrequency ablation

- stereotactic ablative body radiotherapy • stereotactic body radiotherapy 
stereotactic ablative body radiotherapy (SABR) has gained an increasing role in the management of unresectable disease supported largely by (at the time of publication of this manuscript) retrospective and Phase I/II studies that have reported promising local control and survival outcomes [8-11].

The intent of this article is to review the current indications and literature for the evidence in the use of RFA, comparing it with some of the newer and less established ablation techniques. In particular, comparative efficacy based on tumor size will be discussed which, as will become evident, is a critical factor in determining the effectiveness of ablation irrespective of the modality employed. A brief discussion on the technique, mechanisms of action and the relative advantages and potential limitations of each modality will also be incorporated into this review.

\section{Current treatment algorithms}

A number of classification and staging systems currently exist in the management of HCC; the Barcelona Clinic Liver Cancer (BCLC; Figure 1) classification is the most widely endorsed and is used for both clinical management of patients with HCC and also for trial design [12].

The BCLC criteria have been externally validated in European, American as well as Asian patient cohorts [12,14-16] and endorsed by the European Society for the Study of the Liver (EASL) as well as the American Association for the Study of Liver Diseases (AASLD) [17,18]. Within the criteria, ablation is offered to patients with very early (single small tumors $<2 \mathrm{~cm}$ ) or early disease (single or 3 tumors $<3 \mathrm{~cm}$ ) in Childs-Pugh categories A and B, for patients not suitable for resection or transplantation. Although not incorporated in the criteria, ablation is also commonly used as bridging therapy in patients with early disease awaiting transplant.

While not entirely validated in a European population of HCC patients [19], the Hong Kong Liver Cancer (HKLC) staging system (Figure 2), proposed by Yau et al. [20] in 2014 has reportedly demonstrated superiority when compared with the BCLC system in predicting patient prognosis as well as in identifying subsets of patients for more aggressive treatments. In particular, ablation is recommended for appropriate patients with tumors up to $5 \mathrm{~cm}$ (in contradistinction to BCLC). Multifocal tumors or intrahepatic vascular invasion are not necessarily considered as contraindications to surgical management [20]. Given the different approaches to ablation, determination of an optimal strategy requires a more complex approach that considers not only the size, but also geometry and location that may be better suited to one modality over another.

\section{Techniques \& mechanisms of action}

\section{- Thermal ablation}

Thermal ablation, as the name implies, relies on generation of heat within target tissue to achieve tissue destruction. This is governed by a complex set of interactions, which determine the spatial distribution of internal temperatures in target tissue, as illustrated by the bio-heat equation, first laid down by Pennes in the seminal 1948 Journal of Applied Physiology paper [21] and since further refined to the following equation:

pc $\partial \mathrm{T} / \partial \mathrm{t}=\nabla \cdot(\mathrm{k} \nabla \mathrm{T})+\mathrm{q}^{\prime \prime \prime} \mathrm{m}+\boldsymbol{\omega} \mathbf{p b c b}(\mathrm{Ta}-\mathrm{T})$

$\rho=$ mass density $(\mathrm{kg} / \mathrm{m} 3), \mathrm{c}=$ specific heat $(\mathrm{J} / \mathrm{kg} . \mathrm{K}), \mathrm{k}=$ thermal conductivity, $\mathrm{T}=$ tissue

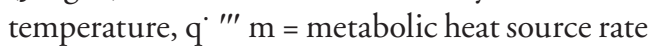
(W/m3), $\Omega$ = perfusion rate, volumetric flow rate of blood per volume of tissue $(\mathrm{s}-1), \mathrm{Ta}=$ arterial blood temperature

While a full analysis of the bioheat equation is beyond the scope of the discussion, what the equation demonstrates is that final target tissue temperatures depends on the density of the tissue as well its thermal conductivity, with loss of temperature due to tissue perfusion (commonly termed the 'heat-sink' effect). The heat-sink effect and rate of heat dissipation from the target tissue has important practical ramifications, as factors such as tumor size and proximity to vessels limit thermal flux, which in turn may limit the size and effectiveness of the RFA ablation zone. MWA, a relatively newer ablative technology, may offer some potential advantages over RFA based on proximity of the target tumor to major intrahepatic vessels and therefore reducing or eliminating the heat-sink effect. Further details regarding the individual technologies will be discussed below.

\section{Radiofrequency ablation}

RFA generates heat from ionic agitation of electrons caused by alternating current in the radiofrequency range of 200-1200 Mhz, transmitted by placing a needle electrode into target tissue either percutaneously or laparoscopically. 


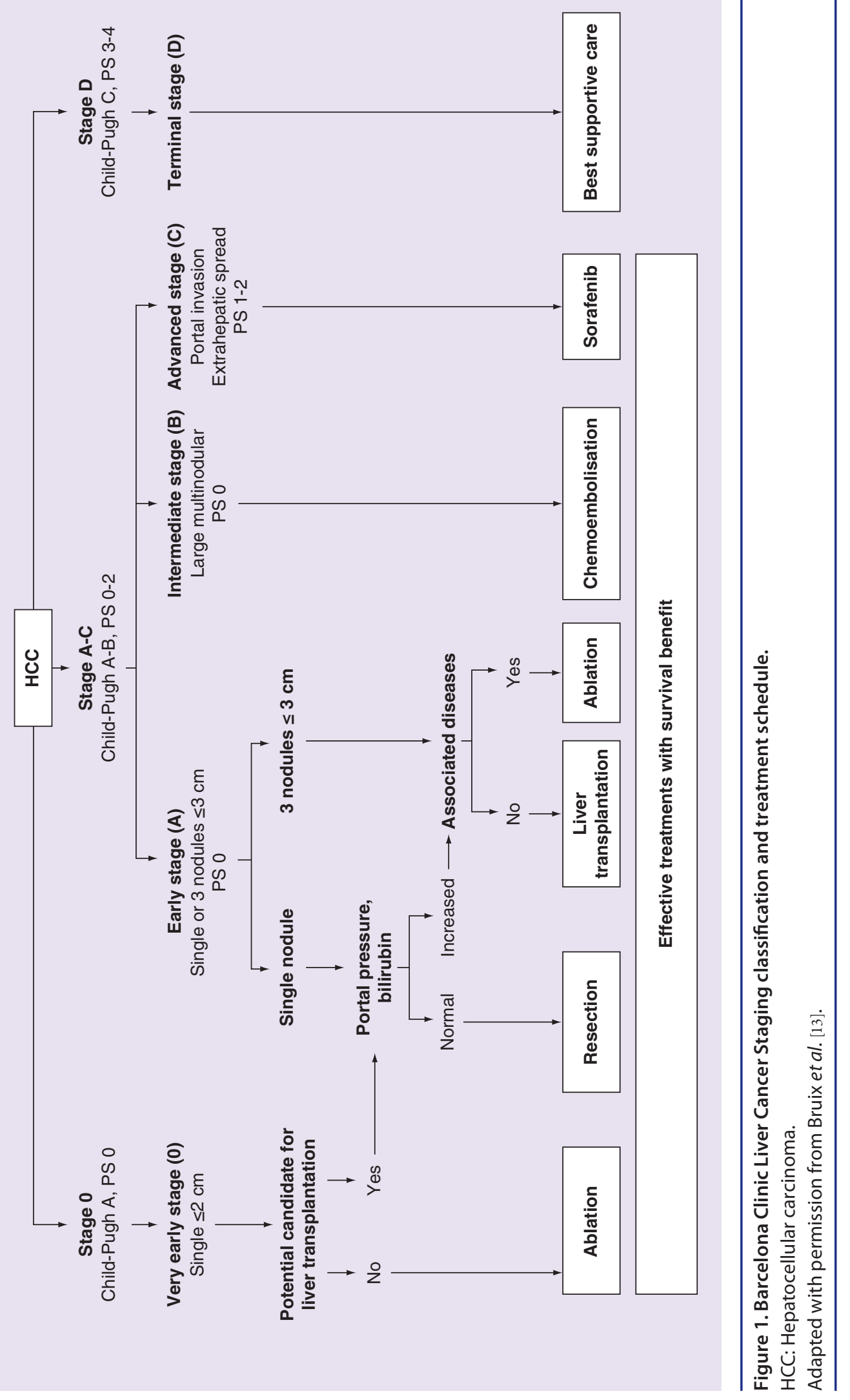



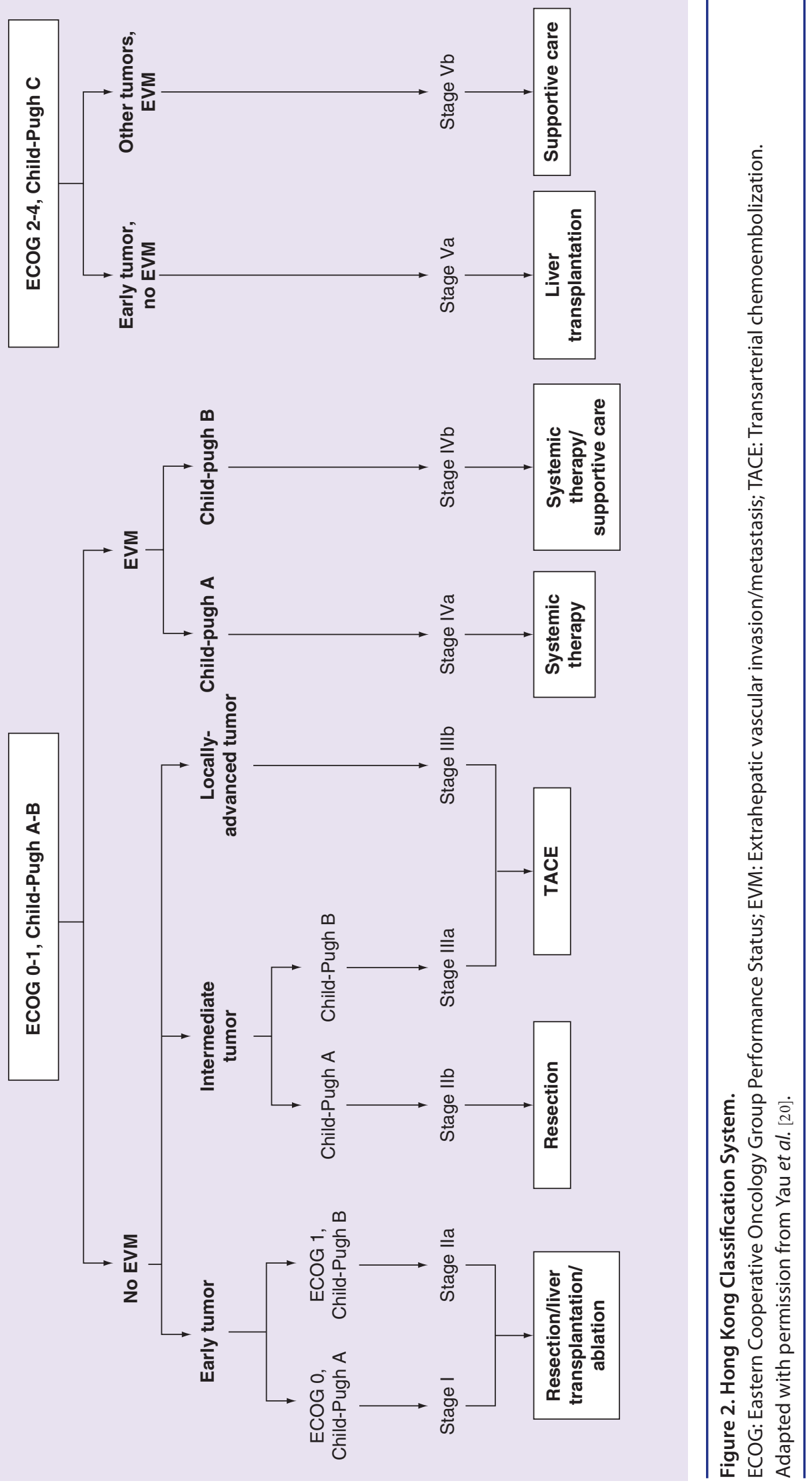
Grounding pads placed on the patients' thighs or back form the complete electrical circuit and provide a larger surface area for heat dissipation. Thus, the electron flux density becomes higher at the electrode (due to significantly smaller surface area as compared with the grounding pad) and as a result, ablative temperatures (target tissue $>60^{\circ} \mathrm{C}$ ) can be obtained via the generation of frictional heat from the oscillation of electrons. Percutaneous approaches may be performed under ultrasound (US) or computed tomography (CT) guidance, either under heavy conscious sedation or general anesthetic. The heat generated leads to destruction of tissue (tumor) by coagulative necrosis; a margin of $0.5-1 \mathrm{~cm}$ around the tumor is usually included to ensure microsatellite tumor destruction. Due to the very nature of RFA, only a few millimeters of tissue surrounding the electrode undergoes active heating, with the remainder of the ablation zone created through thermal conduction $[22,23]$. Therefore, multiple overlapping ablations (with a single electrode or an array of electrodes) may be performed to achieve larger and/or more complex geometries.

As eluded to previously important limitations of RFA include its susceptibility to the heatsink effect, in other words, loss of heat due to adjacent vessels such as portal or hepatic veins, and the dependency on ionic flux that requires hydrated tissues. RFA may also be limited by impedance resulting from tissue destruction, in other words, decreasing thermal conductivity, which is an important component of tissue heat generation [24].

\section{Microwave ablation}

The ultimate mechanism of action of tumor destruction for MWA is the same as RFA, namely thermally induced coagulative necrosis. The heat, however, is generated by high frequency (>900 MHz) electromagnetic energy, via interaction with protons predominantly (but not completely) residing within water molecules, causing them to rapidly flip their electrical charge (hydrogen has a positive charge and oxygen negative) 2-5 billion times a second, which ultimately generates friction and heat [22]. MWA, like RFA, can be performed laparoscopically by hepatobiliary surgeons or percutaneously by interventional radiologists with US or CT guidance and patients under conscious sedation or general anesthetic.

Due to the inherent properties of electromagnetic radiation and the broader fields of power density, MWA is able to generate more uniform and larger ablation zones, particularly when adjacent to large vessels. Furthermore, it is not susceptible to increasing impedance from ablated tissue, and not completely dependent on hydrated tissue, both of which are important limitations for RFA. Lastly, as opposed to RFA, multiple ablations can be performed simultaneously as the method of heat generation is by electromagnetic energy.

\section{- Stereotactic ablative body radiotherapy}

SABR delivers high dose, highly focused external beam radiotherapy over a short time period (3-5 treatment days). It maximizes the potential increased lethal DNA damage seen with larger radiation doses and minimizes tumor proliferation with the shortened treatment schedule. Moreover, in comparison to other forms of external beam radiotherapy, there is better normal tissue sparing and greater patient convenience.

Radiation-induced liver disease has long been the most concerning side effect associated with liver radiation. It is described as classical (on the background of normal liver - anicteric ascites and hepatomegaly) and nonclassical (on the background of a cirrhotic liver - rising serum transaminases and jaundice) and may occur at any time between 2 and 12 weeks post treatment with the majority demonstrating a self-limiting condition, likely due to hepatocyte recovery and regeneration. A minority of patients, however, may go on to develop worsening of their liver function leading to fulminant liver failure. Additional common side effects associated with the use of liver SABR include fatigue, nausea, vomiting, gastritis and liver pain.

During normal respiration, the liver may move as much as $3 \mathrm{~cm}$ [25], in the superior/inferior direction, with elastic compression deforming the liver itself. As a result of this distortion, excessive use of additional margins and dose to adjacent normal tissue may occur. Typical modalities used to limit liver motion include breath hold, respiratory gating and tracking, active breathing control, abdominal compression with 4-dimensional computed tomography (4DCT) and free breathing with 4DCT.

In order to provide real-time navigation to account for motion, three gold seed fiducials are placed by interventional radiologists around the tumor prior to simulation, to aid in tumor localization. Patients are simulated with a triphasic CT scan and with a 4DCT scan. 
Table 1. Results from case series of hepatocellular carcinoma patients treated with radiofrequency ablation.

\begin{tabular}{|c|c|c|c|c|c|c|c|c|}
\hline Study (year) & Number & Device & $\begin{array}{l}\text { Mean F/U } \\
\text { (mo.) }\end{array}$ & $\begin{array}{l}\text { 1-year } \\
\text { OS }\end{array}$ & $\begin{array}{l}\text { 3-year } \\
\text { os }\end{array}$ & $\begin{array}{l}5 \text {-year } \\
\text { OS }\end{array}$ & $\begin{array}{l}10 \text {-year } \\
\text { OS }\end{array}$ & $\begin{array}{l}\text { LTP (\%)/LTP-free } \\
\text { survival (mo.) }\end{array}$ \\
\hline N'Kontchou et al. (2009) [35] & 235 & Covidien Cool-tip & 27 & - & 60 & - & - & $11.5 / 23$ \\
\hline Kim et al. (2013) [33] & 1305 & Covidien Cool-tip (+ 3 others) & 33.4 & 95.5 & 77.9 & 59.7 & 32.3 & $16.4 /-$ \\
\hline Facciorusso et al. (2014) [34] & 103 & Model $1500 \mathrm{~L}, \mathrm{RITA}$ & 78 & 97 & $\begin{array}{l}65(4- \\
\text { year })\end{array}$ & 52 & - & - \\
\hline
\end{tabular}

This is fused with the patient's diagnostic CT or Magnetic resonance imaging (MRI) images (when available). A gross tumor volume is delineated using all available imaging and based on the individual patient's motion management a planning target volume is added. Tumor coverage and total dose is determined based on the irradiated liver volume normal tissue complication probability normogram and organ at risk dose constraints, using volumetric modulated arc therapy (VMAT) conformal arc therapy. Patients are treated every other day with three to five treatments and tumor positioning is assessed by pretreatment based on and matched to the location of fiducials on cone beam CT.

\section{- Irreversible electroporation}

Irreversible electroporation (IRE) is a nascent nonthermal ablation modality, which has been used mainly in the control of unresectable pancreatic carcinoma [26,27]. IRE exploits the electrical gradient that is present across all cell membranes by applying short pulses of very high-voltage direct current energy (90 pulses of $1000-3000 \mathrm{~V} / \mathrm{cm}$ ) leading to the destabilization of the electrical potential across the cell membrane, resulting in the formation of permanent nanopores in the lipid bilayer via involution of the biphospholipid membrane [28]. These nanopores induce tissue death via apoptosis while fully preserving the peri-ablative zone architecture including blood vessels, bile ducts and surrounding normal tissues [29] due to the presence of gap junctions of the smooth muscle cells and higher contents of collagenous/elastic fibrous tissue in bile ducts and blood vessels that act as a barrier preventing the electrical current [28].

IRE is usually performed under CT guidance and due to its mechanism of action, has to be performed under general anesthesia with muscular blockade and pulses timed to the cardiac cycle to prevent arrhythmias. Multiple unipolar or bipolar needle electrodes may be used, with pulses delivered through only one cathode and anode at a time; unipolar leads must also be placed as parallel as possible to achieve a predictable ablation zone [30].

\section{Current indications \& evidence \\ - RFA in early HCC}

In a position statement published in 2009 on the treatment of liver tumors, primarily HCC and colorectal metastases, the Society of Interventional Radiologists concluded that percutaneous RFA was a safe and effective treatment for selected patients [31].

Since the publication of the position statement, further published evidence supporting the use of RFA in early-stage HCC has increased the level and confidence of the evidence. Recently, a 10-year consecutive case series review by Shiina et al. in 2012 [32] concluded that RFA was safe and a locally curative procedure, which could be first-line treatment for selected patients with early HCC and potentially as a substitute for surgical resection of small solitary lesions in patients at risk for recurrence. Overall survival (OS) rates at 5 and 10 years were 60.2 and $27.3 \%$, respectively, with other case series reporting concordant findings and 5-year survival rates ranging from 40 to $67.9 \%$ [33-36] (Table 1).

Local recurrence (LR) rates in multiple studies have, however, demonstrated significant heterogeneity (Table 1); Shiina et al. [32] reported a 5-year LR rate of $3.2 \%$, with multivariate hazard ratios trending toward significance with increasing tumor size $(2.1-3.0 \mathrm{~cm}=1.3$, $3.1-5.0 \mathrm{~cm}=1.29$ and $>5 \mathrm{~cm}=1.25)$, while Kim et al. [33] reported a 5-year LR rate of 27\%, with increasing tumor size the only significant factor on univariate analysis. The heterogeneity in LR data is likely due to differences in HCC etiologies, different approaches to insufficient ablation margins, concurrent use of transarterial 
chemoembolization (TACE), different definitions of tumor recurrence at imaging and variances in patient population [37].

\section{- RFA versus resection}

While resection has been the traditional first-line treatment of choice, depending on the criteria applied (such as multifocality, tumor size and vascular invasion) and comorbidities, as few as $10 \%$ of patients with HCC are suitable for surgery [6]. As RFA has demonstrated efficacy in treating early-stage HCC patients not suitable for resection, a few studies have attempted to compare the two as first-line treatments in a very early disease. In a 2006 randomized controlled trial (RCT) involving 180 patients, Chen et al. [38] demonstrated no statistically significant difference between RFA and surgical resection, with 3- and 4-year OS rates of $71.4 \%, 67.9 \%$ and $73.4 \%, 64 \%$, respectively. There was also no difference in outcomes when subgroup analysis based on tumor size was performed $(<3 \mathrm{~cm}$ and $3.1-5 \mathrm{~cm})$ [38]. The authors concluded in favor of RFA based on decreased cost and complication rates. Two additional RCTs, Feng et al. in 2012 [39] and Fang et al. in 2014 [40], involving 168 and 120 patients, respectively, also demonstrated no statistically significant difference in local control or OS between RFA or resection (Table 2).

However, in a 2010 study involving 230 patients confined to Milan criteria, randomized equally to RFA or surgical resection, 5 -year OS rates were 54.78 and $75.65 \%$, respectively [41], a statistically significant result favoring surgery over RFA. Corresponding 5-year recurrence-free survival and overall recurrence rates for the RFA group and resection group were $28.69 \%, 63.48 \%$ and $51.30 \%, 41.74 \%$, respectively. Further subgroup analysis based on tumor size $(<3 \mathrm{~cm}$ and $3-5 \mathrm{~cm})$ and multifocality similarly demonstrated superiority of resection over RFA [41]. Factors that may have contributed to these findings include the variation in multiplicity and size of tumors that were selected in patients presenting under the Milan criteria.

\section{- RFA versus MWA}

RFA is efficacious in treating early-stage HCC both in patients unsuitable for surgical treatment or even as first-line treatment in suitable patients. The theoretical advantages of MWA have been outlined previously; however, the clinical data require further exploration.

A 2015 meta-analysis by Chinnaratha et al., which included one RCT, one nonrandomized prospective comparison trial and eight retrospective observational studies, demonstrated no difference in overall local tumor progression (LTP) rates or 1- and 3-year OS rates between the two modalities [42]. Subgroup analysis indicated a benefit for MWA for local control in tumors outside of Milan criteria (single tumor $>5 \mathrm{~cm}$ or $>3$ nodules) [42].

A systemic review published in 2016, which included one RCT and six retrospective studies, also demonstrated no statistically significant difference in LR rates between the two modalities (OR: 1.01; 95\% CI 0.53-1.87; p = 0.98) [43]. In a sub-group analysis of 3 studies [44-46], which included larger tumors, MWA significantly outperformed RFA in LTP rates (OR: 0.46; 95\% CI: $0.24-0.80 ; p=0.02$ ) [43]. Therefore, while there are no significant overall differences in LTP and OS rates between the two modalities, published data favor MWA for local control in larger tumors.

\section{- SABR in early HCC}

Undoubtedly, a growing interest has developed with SABR in the management of HCC for curative intent with only a few small prospective Phase I/II studies that have reported on local control, survival outcomes and toxicity $[47,48]$. There have also been several retrospective single institutional studies including those by Sanuki et al. [49] detailing outcomes of 185 HCC patients treated with SABR for tumors

Table 2. Results of randomized control trials comparing radiofrequency ablation and resection in early hepatocellular carcinoma.

\begin{tabular}{|llllllll|}
\hline Study (year) & Number & $\begin{array}{l}\text { RFA 1-year } \\
\text { OS (\%) }\end{array}$ & $\begin{array}{l}\text { Resection } \\
\text { 1-year OS (\%) }\end{array}$ & $\begin{array}{l}\text { RFA 3-year OS } \\
\text { (\%) }\end{array}$ & $\begin{array}{l}\text { Resection } \\
\text { 3-year OS (\%) }\end{array}$ & $\begin{array}{l}\text { RFA 3-year } \\
\text { DFS (\%) }\end{array}$ & $\begin{array}{l}\text { Resection 3-year } \\
\text { DFS (\%) }\end{array}$ \\
\hline Chen et al. (2006) [38] & 180 & 95.8 & 93.3 & 71.4 & 73.4 & 64.1 & 69 \\
\hline Huang et al. (2010) [41] & 230 & 86.96 & 98.2 & 69.57 & 92.17 & 46.08 & 60.87 \\
\hline Feng et al. (2012) [39] & 168 & 93.1 & 96 & 67.2 & 74.8 & 49.6 & 61.1 \\
\hline Fang et al. (2014) [40] & 120 & 97.5 & 93.7 & 82.5 & 77.5 & 55.4 & 41.3 \\
\hline DFS: Disease-free survival; OS: Overall survival; RFA: Radiofrequency ablation. & & & & \\
\hline
\end{tabular}


$\leq 5 \mathrm{~cm}$. The 3-year local control and OS rates were 91 and $70 \%$, respectively and only $13 \%$ of patients had a $\geq$ grade 3 acute toxicity. A group from Korea [50] published on $82 \mathrm{HCC}$ patients with a median tumor size of $3 \mathrm{~cm}(1-7 \mathrm{~cm})$ who received a median dose of $51 \mathrm{~Gy}$ in 3 fractions with reported 2 -year local control and OS rates of $87 \%$ and $63 \%$ respectively.

Yoon et al. [11] described reducing local control rates with SBRT as tumor size increased (LC at 3 years $100 \%<2 \mathrm{~cm}$ and $76 \%>3 \mathrm{~cm}$ ) and Kwon et al. [8] demonstrated increased tumor progression rates in tumors greater than $32 \mathrm{cc}$ (corresponding to approximately $4 \mathrm{~cm}$ ). Currently, the standard of care in patients who are not candidates for surgery or local thermal ablative therapies due to multiplicity and/ or tumor volume is the noncurative option of TACE. The use of TACE with and without SABR has been reported [51] in patients with large unresectable HCCs, describing promising response and OS rates in the combined group (2-year survival $36.8 \%$ vs $14.3 \%$ ). More data are required to further elucidate the role of SABR alone or in combination as a potential ablative therapy in the larger unresectable HCC population.

\section{- Thermal ablation versus SABR}

As of the time of publication, no randomized prospective data directly comparing thermal ablation to SABR in the ablative setting has been conducted. As previously described, limitations to the use of thermal ablative therapies include location and size of tumors. SABR has the theoretical ability to overcome technicalities such as location by developing individualized patient plans and using highly focused beam arrangements, assuming that control of motion artifact and adequate dose planning results in minimal nontargeted irradiation to normal liver parenchyma and adjacent critical tissues (such as the stomach, gastrointestinal tract and lung).

Wahl et al. [9] retrospectively compared RFA to SABR in patients with early-stage, liverconfined HCC. General tumor characteristics were similar between groups and median tumor size was 1.8 (RFA) versus $2.2 \mathrm{~cm}$ (SABR) and overall there were similar local control and survival outcomes, with excellent side-effect profiles described. The study found no statistically significant difference in freedom from local progression between groups for tumors less than $2 \mathrm{~cm}$ (HR: 2.50 ; 95\% CI: 0.72-8.67; $\mathrm{p}=0.15$ ); however, in tumors greater than $2 \mathrm{~cm}$ SABR was associated with a significantly improved freedom from local progression (HR: 3.35; 95\% CI: 1.17-9.62; $\mathrm{p}=0.025)$. The authors concluded that both treatment modalities are effective options in the early inoperable setting and SABR may be a reasonable first-line option in treating the larger, greater than $2 \mathrm{~cm}$, inoperable tumors. MWA, IRE and complex geometry multielectrode RFA were not incorporated into the retrospective analysis.

In a recent paper comparing the cost-effectiveness of SABR versus RFA in treating HCC, Pollom et al. [52] developed a decision-analytic Markov model for patients with inoperable, localized HCC who were eligible for both treatment modalities. They concluded that a treatment strategy which consisted of RFA as initial treatment followed by SBRT as salvage therapy (RFA-SBRT) was the most efficacious and cost effective as compared with RFA-RFA and SBRT-RFA strategies.

\section{- Irreversible electroporation}

IRE has mainly been used in the treatment of locally advanced pancreatic adenocarcinoma [26,27]; its role in treating liver tumors, particularly HCC, is less certain, with no long-term survival data available. A prospective cohort study containing the largest number of HCC patients treated with IRE to date included a total of 25 patients with 48 tumors, 22 of which were HCCs with a median tumor size of $4.6 \mathrm{~cm}$ [53]. LR rates were significantly associated with tumor size, with LR rate of $9.7 \%$ for tumors $<5 \mathrm{~cm}$ and $64.7 \%$ if $>5 \mathrm{~cm}$, with HCC histology being an additional associated variable (as compared with other primary or nonprimary liver tumors).

Other published cohort studies, which have included a relatively large number of patients with liver tumors, do not contain a significant number of patients with HCC (2-14 patients) [54-56]. Cannon et al. included 14 patients with HCC (median size $2.1 \mathrm{~cm}: 1.3-4.5 \mathrm{~cm}$ ) and demonstrated LR-free survival rates of 90 and $50 \%$ at 6 and 12 months, respectively [55]. Thompson et al. included ten HCC patients with 18 tumors, demonstrating complete response in 15/18 tumors at 3 months follow-up and no response in tumors greater than $5 \mathrm{~cm}$ [56]. Analysis of the remainder of the studies demonstrates relatively poor local control as compared with thermal ablation; the utility of IRE may therefore lie in treating 
patients who are not suitable for resection, ablation or radiotherapy. No long-term follow-up data vis-a-vis LR rates, progression-free survival and OS have been reported.

\section{Conclusion}

As we have seen, there are a number of local ablative therapies available for the treatment of early or unresectable HCC. Thermal ablative modalities, mainly RFA, have not only demonstrated their efficacy in treating early HCC, but also have proved to be as effective as surgical resection in the treatment of early uncomplicated disease in selected patients, with similar LTP-free and OS rates; results obtained with less morbidity. MWA, while not demonstrating

\section{EXECUTIVE SUMMARY}

\section{Background}

- Primary liver cancer, mainly hepatocellular carcinoma (HCC), is one of the most common malignancies in the world.

- Surgical management, either resection or transplantation, are definite curative therapies but less than one in five patients are suitable candidates. Local ablative modalities, which may be curative in intent, are alternative treatments.

\section{Thermal ablation}

- Thermal ablation techniques operate by generation of heat in target tissue, either by electrical (radiofrequency ablation, RFA) or electromagnetic energy (microwave ablation, MWA), resulting in protein denaturing and coagulative necrosis.

- RFA is the traditional thermal ablative modality, with a substantial supporting body of evidence, demonstrating efficacy in treating early-stage HCC; 5 -year overall survival rates across multiple studies range from 40 to $67.9 \%$. RFA can also be considered as first-line treatment (vs surgical resection) in selected patients with uncomplicated early-stage disease.

- MWA is the more recent thermal ablation technique, with similar efficacy and overall survival to RFA in treating early HCC. Due to its mechanism of action, it may provide better local control in larger and perivascular tumors.

\section{Stereotactic ablative body radiotherapy}

- Stereotactic ablative body radiotherapy (SABR) is at present generally reserved for tumors unsuitable for thermal ablation by virtue of location and/or size or as salvage therapy after other locoregional therapies.

- Recent studies have, however, demonstrated promising results in treating early HCC, with 3-year local control and overall survival rates of 91 and $70 \%$ in a retrospective study of 185 patients. A number of Phase I/II trials have also demonstrated encouraging results.

\section{Irreversible electroporation}

- Irreversible electroporation (IRE) is a nonthermal ablative modality that relies on disrupting the electrical gradient across cell membranes by application of short pulses of high-voltage direct current energy. This creates permanent nanopores, leading to cell death via apoptosis (as opposed to thermally induced coagulative necrosis).

- It has mainly been used, with some success, in the treatment of locally advanced pancreatic adenocarcinoma. Its role in treating HCC is less established but currently under investigation; its utility, so far, may lie in treating patients who are unsuitable for resection, thermal ablation or other forms of locoregional therapies.

\section{Conclusion}

- Thermal ablative treatments, mainly RFA, supported by a substantial body of evidence, have demonstrated efficacy in treating early-stage HCC and may be considered as first-line therapy in selected patients with early uncomplicated disease; MWA offers potentially better local control in larger and perivascular tumors.

- Due to refinements in SABR technique, there are emerging data supporting its increasing use in treating early or unresectable HCC. It is currently used as salvage therapy or in patients unsuitable for traditional forms of locoregional treatments.

- Nascent technologies such as IRE may provide additional options for disease control in patients who are either unsuitable for or have exhausted traditional avenues of surgical or ablative treatments, although current evidence do not support their widespread use. 
overall superiority to RFA despite its theoretical advantages, has nevertheless reported results suggesting the modality to be more effective in treating perivascular and larger tumors. While there is a paucity of prospective and RCTs evaluating SABR in treating unresectable HCC, a number of recent studies have demonstrated promising results. Furthermore, with relatively nascent and emerging technologies such as IRE supported by a body of growing evidence, the precision and progress that has been achieved has led to expanding indications and roles for various modalities.

Indications for use of RFA within our institution generally include solitary lesions of $<3 \mathrm{~cm}$ without contact with a vascular structure with simple geometries that are conducive to a single electrode placement. Indications for the use of MWA include lesions ranging from 3 to $4.5 \mathrm{~cm}$ in size, and lesions that are near vascular structures. Additional consideration is made in the setting where the desiccating properties of MWA may help 'pull the tumor away' from tissue planes that may represent surgical resection margins in the future, or in subcapsular lesions at risk to rupture during RFA. Indications for the use of SABR vary between centers and are often reserved for patients who are ineligible for all other treatment modalities (i.e., surgery, RFA, MWA and TACE) due to issues related to liver function, medical comorbidities, location, age and size.

\section{Future perspective}

The improvements in our understanding of the biology and nature of HCC, married with newer nonsurgical techniques to cure, downstage and bridge patients to transplantation has created a veritable cornucopia of options for therapy. As HCC represents the end point of chronic inflammatory conditions (such as alcohol induced liver disease, viral hepatitis, chronic inflammatory states and genetic disorders), metachronous disease presentation is the normal, as opposed to exceptional, situation. Therefore, many patients often undergo serial treatments ranging from surgery to ablation and to embolization. With a maturing body of literature and the evolution to multidisciplinary tumor boards, local expertise supplemented by the increasing body of data will serve to address and acknowledge the noncompetitive nature and role of contemporary therapies for the treatment of the HCC patient.

\section{Financial \& competing interests disclosure}

The authors have no relevant affiliations or financial involvement with any organization or entity with a financial interest in or financial conflict with the subject matter or materials discussed in the manuscript. This includes employment, consultancies, honoraria, stock ownership or options, expert testimony, grants or patents received or pending, or royalties.

No writing assistance was utilized in the production of this manuscript.

\section{Open access}

This article is distributed under the terms of the Creative Commons Attribution License 3.0 which permits any use, distribution, and reproduction in any medium, provided the original author(s) and the source are credited. To view a copy of the license, visit http://creativecommons.org/ licenses/by/3.0/

\section{References}

Papers of special note have been highlighted as: - of interest; $\bullet$ of considerable interest

1 Ferlay J, Shin HR, Bray F, Forman D, Mathers C, Parkin DM. Estimates of worldwide burden of cancer in 2008: GLOBOCAN 2008. Int. J. Cancer 127(12), 2893-2917 (2010).

2 Torre LA, Bray F, Siegel RL, Ferlay J, Lortet-Tieulent J, Jemal A. Global cancer statistics, 2012. CA Cancer J. Clin. 65(2), 87-108 (2015).

3 Yau T, Chan P, Epstein R, Poon RT. Management of advanced hepatocellular carcinoma in the era of targeted therapy. Liver Int. 29 (1), 10-17 (2009).
4 Mazzaferro V, Bhoori S, Sposito C et al. Milan criteria in liver transplantation for hepatocellular carcinoma: an evidence-based analysis of 15 years of experience. Liver Transpl. 17(Suppl. 2), S44-S57 (2011).

- Systemic analysis analyzing influence of Milan criteria in post-transplantation survival.

5 European Association for the Study of The L, European Organisation For R, Treatment Of C. EASL-EORTC clinical practice guidelines: management of hepatocellular carcinoma. J. Hepatol. 56(4), 908-943 (2012).

6 Forner A, Llovet JM, Bruix J. Hepatocellular carcinoma. Lancet 379(9822), 1245-1255 (2012).
7 Llovet JM, Fuster J, Bruix J. Intention-to-treat analysis of surgical treatment for early hepatocellular carcinoma: resection versus transplantation. Hepatology 30(6), 1434-1440 (1999).

8 Kwon JH, Bae SH, Kim JY et al. Long-term effect of stereotactic body radiation therapy for primary hepatocellular carcinoma ineligible for local ablation therapy or surgical resection. Stereotactic radiotherapy for liver cancer. BMC Cancer 10, 475-475 (2010).

9 Wahl DR, Stenmark MH, Tao Y et al. Outcomes after stereotactic body radiotherapy or radiofrequency ablation for hepatocellular carcinoma. J. Clin. Oncol. 34(5), 452-459 (2015). 
10 Bujold A, Massey CA, Kim JJ et al. Sequential Phase I and II trials of stereotactic body radiotherapy for locally advanced hepatocellular carcinoma. J. Clin. Oncol. 31(13), 1631-1639 (2013).

11 Yoon SM, Lim Y-S, Park MJ et al. Stereotactic body radiation therapy as an alternative treatment for small hepatocellular carcinoma. PLoS ONE 8(11), e79854 (2013).

12 Llovet JM, Di Bisceglie AM, Bruix J et al. Design and endpoints of clinical trials in hepatocellular carcinoma. J. Natl Cancer Inst. 100(10), 698-711 (2008).

13 Bruix J, Reig M, Sherman M. Evidence-based diagnosis, staging, and treatment of patients with hepatocellular carcinoma. Gastroenterology 150 (4), 835-853 (2016).

14 Marrero JA, Fontana RJ, Barrat A et al. Prognosis of hepatocellular carcinoma: comparison of 7 staging systems in an American cohort. Hepatology 41(4), 707-716 (2005).

- Comparison of multiple staging systems in an American cohort; Barcelona Clinic Liver Cancer provided the best prognostic stratification.

15 Cillo U, Vitale A, Grigoletto F et al. Prospective validation of the Barcelona Clinic Liver Cancer staging system. J. Hepatol. 44(4), 723-731 (2006).

16 Hsu CY, Liu PH, Hsia CY et al. Nomogram of the Barcelona Clinic Liver Cancer system for individual prognostic prediction in hepatocellular carcinoma. Liver Int. 36(10), 1498-1506 (2016).

17 Bruix J, Sherman M, Practice Guidelines Committee AaFTSOLD. Management of hepatocellular carcinoma. Hepatology 42(5), 1208-1236 (2005).

18 Bruix J, Sherman M, Llovet JM et al. Clinical management of hepatocellular carcinoma. Conclusions of the Barcelona-2000 EASL conference. European Association for the Study of the Liver. J. Hepatol. 35(3), 421-430 (2001).

19 Adhoute X, Penaranda G, Bronowicki JP, Raoul JL. Usefulness of the HKLC vs. the BCLC staging system in a European HCC cohort. J. Hepatol. 62 (2), 492-493 (2015).

20 Yau T, Tang VY, Yao TJ, Fan ST, Lo CM, Poon RT. Development of Hong Kong Liver Cancer staging system with treatment stratification for patients with hepatocellular carcinoma. Gastroenterology 146(7), 1691-1700 e1693 (2014).

21 Pennes HH. Analysis of tissue and arterial blood temperatures in the resting human forearm. J. Appl. Physiol. 1(2), 93-122 (1948).
22 Simon CJ, Dupuy DE, Mayo-Smith WW. Microwave ablation: principles and applications. Radiographics 25(Suppl. 1), S69-S83 (2005).

23 Organ LW. Electrophysiologic principles of radiofrequency lesion making. Appl. Neurophysiol. 39(2), 69-76 (1976).

24 Goldberg SN, Gazelle GS, Solbiati L, Rittman WJ, Mueller PR. Radiofrequency tissue ablation: increased lesion diameter with a perfusion electrode. Acad. Radiol. 3(8), 636-644 (1996).

25 Langen KM, Jones DT. Organ motion and its management. Int. J. Radiat. Oncol. Biol. Phys. 50(1), 265-278 (2001).

26 Narayanan G, Hosein PJ, Arora G et al. Percutaneous irreversible electroporation for downstaging and control of unresectable pancreatic adenocarcinoma. J. Vasc. Interv. Radiol. 23(12), 1613-1621 (2012).

27 Martin RC, 2nd, Mcfarland K, Ellis S, Velanovich V. Irreversible electroporation therapy in the management of locally advanced pancreatic adenocarcinoma. J. Am. Coll. Surg. 215(3), 361-369 (2012).

28 Yu H, Burke CT. Comparison of percutaneous ablation technologies in the treatment of malignant liver tumors. Semin Int. Radiol. 31(2), 129-137 (2014).

29 Lee EW, Chen C, Prieto VE, Dry SM, Loh CT, Kee ST. Advanced hepatic ablation technique for creating complete cell death: irreversible electroporation. Radiology 255(2), 426-433 (2010).

30 Silk M, Tahour D, Srimathveeravalli G, Solomon SB, Thornton RH. The state of irreversible electroporation in interventional oncology. Semin. Int. Radiol. 31(2), 111-117 (2014).

31 Gervais DA, Goldberg SN, Brown DB et al. Society of Interventional Radiology position statement on percutaneous radiofrequency ablation for the treatment of liver tumors. J. Vasc. Interv. Radiol. 20(1), 3-8 (2009).

- Society of Interventional Radiology position statement supporting the use of radiofrequency ablation in treating liver tumors, including hepatocellular carcinoma (HCC).

32 Shiina S, Tateishi R, Arano T et al. Radiofrequency ablation for hepatocellular carcinoma: 10-year outcome and prognostic factors. Am. J. Gastroenterol. 107(4), 569-577; quiz 578 (2012).

33 Kim YS, Lim HK, Rhim H et al. Ten-year outcomes of percutaneous radiofrequency ablation as first-line therapy of early hepatocellular carcinoma: analysis of prognostic factors. J. Hepatol. 58(1), 89-97 (2013).

34 Facciorusso A, Del Prete V, Antonino M et al. Post-recurrence survival in hepatocellular carcinoma after percutaneous radiofrequency ablation. Dig. Liver Dis. 46(11), 1014-1019 (2014).

35 N'kontchou G, Mahamoudi A, Aout M et al. Radiofrequency ablation of hepatocellular carcinoma: long-term results and prognostic factors in 235 Western patients with cirrhosis. Hepatology 50(5), 1475-1483 (2009).

36 Lee DH, Lee JM, Lee JY et al. Radiofrequency ablation of hepatocellular carcinoma as first-line treatment: long-term results and prognostic factors in 162 patients with cirrhosis. Radiology 270(3), 900-909 (2014).

37 Facciorusso A, Serviddio G, Muscatiello N Local ablative treatments for hepatocellular carcinoma: an updated review. World J. Gastrointest. Pharmacol. Ther. 7(4), 477-489 (2016).

38 Chen MS, Li JQ, Zheng Y et al. A prospective randomized trial comparing percutaneous local ablative therapy and partial hepatectomy for small hepatocellular carcinoma. Ann. Surg. 243(3), 321-328 (2006).

39 Feng K, Yan J, Li X et al. A randomized controlled trial of radiofrequency ablation and surgical resection in the treatment of small hepatocellular carcinoma. J. Hepatol. 57(4), 794-802 (2012).

40 Fang Y, Chen W, Liang X et al. Comparison of long-term effectiveness and complications of radiofrequency ablation with hepatectomy for small hepatocellular carcinoma. J. Gastroenterol. Hepatol. 29(1), 193-200 (2014).

41 Huang J, Yan L, Cheng Z et al. A randomized trial comparing radiofrequency ablation and surgical resection for HCC conforming to the Milan criteria. Ann. Surg. 252(6), 903-912 (2010).

42 Chinnaratha MA, Chuang MY, Fraser RJ, Woodman RJ, Wigg AJ. Percutaneous thermal ablation for primary hepatocellular carcinoma: a systematic review and meta-analysis. J. Gastroenterol. Hepatol. 31(2), 294-301 (2016).

43 Facciorusso A, Di Maso M, Muscatiello N. Microwave ablation versus radiofrequency ablation for the treatment of hepatocellular carcinoma: a systematic review and meta-analysis. Int. J. Hyperthermia 32(3), 339-344 (2016).

- Meta-analysis of literature comparing radiofrequency ablation versus microwave ablation in treating HCC. 
44 Lu MD, Xu HX, Xie XY et al. Percutaneous microwave and radiofrequency ablation for hepatocellular carcinoma: a retrospective comparative study. J. Gastroenterol. 40(11), 1054-1060 (2005).

45 Abdelaziz AO, Nabeel MM, Elbaz TM et al. Microwave ablation versus transarterial chemoembolization in large hepatocellular carcinoma: prospective analysis. Scand. J. Gastroenterol. 50(4), 479-484 (2015).

46 Vogl TJ, Farshid P, Naguib NN et al. Ablation therapy of hepatocellular carcinoma: a comparative study between radiofrequency and microwave ablation. Abdom. Imaging 40(6), 1829-1837 (2015).

47 Mendez Romero A, Wunderink W, Hussain SM et al. Stereotactic body radiation therapy for primary and metastatic liver tumors: a single institution Phase I-II study. Acta Oncol. 45(7), 831-837 (2006).

48 Weiner AA, Olsen J, Ma D et al. Stereotactic body radiotherapy for primary hepatic malignancies - report of a Phase I/II institutional study. Radiother. Oncol. 121(1), 79-85 (2016).
- Phase I/II study of stereotactic ablative body radiotherapy in treating $\mathrm{HCC}$ and cholangiocarcinoma.

49 Sanuki N, Takeda A, Oku Y et al. Stereotactic body radiotherapy for small hepatocellular carcinoma: a retrospective outcome analysis in 185 patients. Acta Oncol. 53(3), 399-404 (2014).

50 Jang WI, Kim MS, Bae SH et al. High-dose stereotactic body radiotherapy correlates increased local control and overall survival in patients with inoperable hepatocellular carcinoma. Radiat. Oncol. 8, 250 (2013).

51 Shim SJ, Seong J, Han KH, Chon CY, Suh $\mathrm{CO}$, Lee JT. Local radiotherapy as a complement to incomplete transcatheter arterial chemoembolization in locally advanced hepatocellular carcinoma. Liver Int. 25(6), 1189-1196 (2005)

52 Pollom EL, Lee K, Durkee BY et al. Cost-effectiveness of stereotactic body radiation therapy versus radiofrequency ablation for hepatocellular carcinoma: a Markov modeling study. Radiology doi:10.1148/radiol.2016161509 161509 (2017) (Epub ahead of print).

53 Niessen C, Igl J, Pregler B et al. Factors associated with short-term local recurrence of liver cancer after percutaneous ablation using irreversible electroporation: a prospective single-center study. J. Vasc. Interv. Radiol. 26(5), 694-702 (2015).

54 Kingham TP, Karkar AM, D’angelica MI et al. Ablation of perivascular hepatic malignant tumors with irreversible electroporation. J. Am. Coll. Surg. 215(3), 379-387 (2012).

55 Cannon R, Ellis S, Hayes D, Narayanan G, Martin RC, 2nd. Safety and early efficacy of irreversible electroporation for hepatic tumors in proximity to vital structures. J. Surg. Oncol. 107(5), 544-549 (2013).

56 Thomson KR, Cheung W, Ellis SJ et al. Investigation of the safety of irreversible electroporation in humans. J. Vasc. Interv. Radiol. 22(5), 611-621 (2011). 